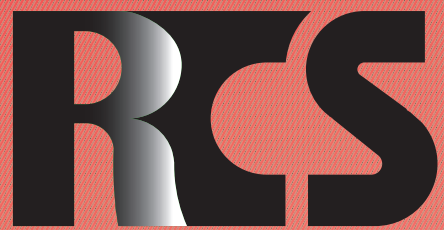

Depósito legal ppi $201502 Z U 4662$

Esta publicación científica en formato digital es continuidad de la revista impresa Depósito Legal: pp $197402 Z U 789$

- ISSN: 1315-9518 • ISSN-E: 2477-9431

Revista de Ciencias Sociales

Universidad del Zulia. Revista de la Facultad de Ciencias Económicas y Sociales Vol. XXVII. No. 2

Abril-Junio 2021

Esta publicación científica en formato digital es continuidad de la revista impresa Depósito Legal: pp $197402 Z$ Z789 ISSN: 1315-9518 


\title{
Desarrollo local en el municipio de Corinto en Cauca-Colombia en el marco del posconflicto
}

\author{
Burbano Vallejo, Edy Lorena* \\ Vargas, Julian**
}

\section{Resumen}

Actualmente, en el territorio colombiano y posterior a la firma del acuerdo de paz firmado con el grupo armado de las FARC, se ha generado esperanza e incertidumbre en regiones que sufrieron el conflicto armado por más de 70 años. En ese sentido, el presente estudio se desarrolló en el municipio de Corinto Cauca, en el marco del Acuerdo de Paz que se firmó en el año 2016, con el fin de analizar la perspectiva de desarrollo local, teniendo en cuenta el reconocimiento del territorio a partir de las dimensiones económica, social, administrativa, cultural y medioambiental, desde un enfoque prospectivo y la aplicación del método de análisis estructural, el cual permite la identificación de variables determinantes del sistema que se establecen por medio de la matriz de influencia - dependencia. De acuerdo con el análisis de la región de Corinto, como principales resultados se tiene que la administración municipal considera el turismo como principal motor del desarrollo del municipio. Por lo cual se concluye, que el gobierno debe invertir en infraestructura física y social con la finalidad de generar el mejor escenario para Corinto en el posconflicto.

Palabras clave: Desarrollo local; Acuerdo de Paz; posconflicto; turismo; Región del Cauca-Colombia.

\footnotetext{
* Doctor en Ciencias Económicas. Magister en Economía Aplicada. Economista. Docente del Programa de Economía de la Universidad de San Buenaventura Cali, Colombia. Integrante del Grupo de investigación en Economía, Gestión, Territorio y Desarrollo Sostenible GEOS. E-mail: elburban@usbcali.edu.co iD ORCID: https://orcid.org/0000-0002$\underline{4366-9172}$

** Administrador de Negocios de la Universidad de San Buenaventura Cali, Colombia. E-mail: julian.vargass4520@ gmail.com (iD) ORCID: https://orcid.org/0000-0002-9081-2285
} 


\title{
Local development in the municipality of Corinto in Cauca-Colombia in the post- conflict framework
}

\begin{abstract}
Currently, in Colombian territory and after the signing of the peace agreement signed with the FARC armed group, hope and uncertainty have been generated in regions that suffered from the armed conflict for more than 70 years. In this sense, this study was developed in the municipality of Corinto - Cauca, within the framework of the Peace Agreement that was signed in 2016, in order to analyze the local development perspective, taking into account the recognition of the territory From the economic, social, administrative, cultural and environmental dimensions, from a prospective approach and the application of the structural analysis method, which allows the identification of determining variables of the system that are established through the influence-dependency matrix . According to the analysis of the Corinto region, the main results are that the municipal administration considers tourism as the main driver of the development of the municipality. Therefore, it is concluded that the government must invest in physical and social infrastructure in order to generate the best scenario for Corinto in the post-conflict situatio.
\end{abstract}

Keywords: Local development; Peace Agreement; post-conflict; tourism; Cauca-Colombia Region

\section{Introducción}

Actualmente, en el territorio colombiano y posterior a la firma del acuerdo de paz firmado con el grupo armado de las FARC, dado que: "El siglo XX y la etapa inicial del siglo XXI en Colombia han transcurrido en un contexto de conflictividad política y social que ha causado profundas heridas en la sociedad colombiana" (Villa y Berrocal, 2019, p.254), se ha generado esperanza e incertidumbre en regiones que sufrieron el conflicto armado por más de 70 años. En dichas regiones el sustento económico se realizaba en medio del conflicto y el temor social, este es el caso del municipio de Corinto, que históricamente ha sido considerado como un territorio estratégico para el desarrollo del conflicto armado, en el cual tuvo presencia el sexto frente de las FARC y la columna móvil Jacobo Arenas.
Según los datos reportados en el Departamento Nacional de Planeación (DNP, 2017), entre el periodo de 1984 a 2017, en el municipio se reportaron 7.386 personas desplazadas o expulsadas del municipio; 2.649 individuos desplazados recibidos del área de influencia y un $44 \%$ de personas secuestradas en el municipio (ver Gráfico I); lo anterior se complementa con el diagnóstico que se realizó para el Plan de Desarrollo Municipal "Nuevas Ideas para la Paz" (2016-2019), en el que se determinó que un $65 \%$ de la población se vio afectada por el conflicto armado. Asimismo, que la región ha tenido un incremento en la siembra y la transformación de cultivos ilícitos (Concejo Municipal de Corinto - Cauca, 2016); Corinto, fue un enclave productivo, puesto que ha tenido precios más altos frente a otras zonas de la misma región. 
Número acumulado de personas desplazadas recibidas

Número acumulado de personas desplazadas expulsadas

Número acumulado de personas secuestradas

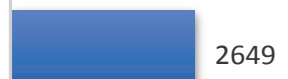

7386

44

En el periodo de 1984-2017

Fuente: Elaboración propia, 2020 a partir de datos del DNP (2017).

\section{Gráfico I: Número de personas desplazadas recibidas y expulsadas, y personas secuestradas (1984-2017)}

De tal manera, se logra evidenciar que la sociedad ha sido orientada a este estilo cultural de vida y a la creación de la conciencia del "dinero fácil"; según Santos (2018), lo que produce un detrimento en las raíces culturales innatas del territorio y, además, se convierte en una amenaza para la conservación de la biodiversidad. Esto ha tenido implicaciones violentas, como la realización de actos terroristas en la región y los desplazamientos, siendo el año 2013 el más negativo para la población, debido a ataques de grupos al margen de la ley. En ese sentido, en el registro Único de Víctimas se reconocen a 7.003 víctimas de la violencia en el municipio, por razones de conflicto armado, como se muestra en el Gráfico I.

Durante más de dos décadas, la región se enfrentó a la incertidumbre y el estigma de ser considerado un municipio de zona roja, además del temor de la población. El tejido empresarial y la administración pública tienen como reto después del 2016, con la firma del Acuerdo de Paz y el inicio del posconflicto, pasar de un territorio de violencia a un territorio de paz, en donde la población se convierta en el principal actor de este cambio, y es allí donde el concepto de desarrollo económico local toma importancia desde su objetivo, que es la búsqueda del mejoramiento de la calidad de vida de la región, "con la explotación de recursos que sean locales y potenciales para así incorporarlos a las diferentes oportunidades en el resto del mundo" (Proaño, et al., 2019, p.85); aunado a ello, esto va en línea con los planteamientos del Estado colombiano formulados en la década de los 90 con la descentralización, pasando los municipios a ser autónomos y a partir de su realidad tomar sus propias decisiones.

En ese orden de ideas, Silva y Sandoval (2012) afirmaron que se debe contar con una "planificación territorial que permita a los gobiernos y comunidad local, ayudar a 
definir sus líneas de acción en el marco de un proceso de desarrollo participativo e integral" (p.9); igualmente, como lo plantearon Boisier (2004) y Alburquerque (2005), el desarrollo local se convierte en una estrategia regional, en la cual deben participar todos los actores involucrados en el territorio, los cuales son la administración pública, las empresas y la comunidad en general, que inciden en las decisiones del territorio. De acuerdo con Fithri, et al. (2020), este desarrollo local permite determinar el potencial de recursos del territorio y sus necesidades, con base en su realidad.

Del mismo modo, Silva y Sandoval (2012) plantearon que los territorios deben formular estrategias de desarrollo local, las cuales deben estar integradas por dos aspectos: La determinación de los objetivos estratégicos y la creación de las condiciones generales, que al mismo tiempo deben ir acompañadas por la administración pública, y que esta realice un constante monitoreo.

Esto permite, en una primera instancia, reconocer al territorio en todas sus dimensiones e identificar el potencial de sus recursos, como estableció Alburquerque (2004); lo que va ir acompañado con un proceso de transformación de la economía y de la sociedad local, a fin de mejorar la calidad de vida de los habitantes por medio de agentes socioeconómicos locales (públicos y privados), cuya actividad busca un uso más eficiente y sustentable de los recursos. $\mathrm{Al}$ respecto, sostiene Delgado (2018) que: ...siendo un territorio una comunidad de intereses donde existen diversas visiones del futuro y los caminos a lograr ese futuro deseado, el pensamiento y la acción de los actores locales, los espacios de diálogo intersubjetivo y las redes de interconexión de las comunidades e instituciones involucradas en el desarrollo territorial son clave para construir en consenso la visión compartida de la transformación. (p.85)

En ese orden de ideas, autores como Alderete y Bacic (2016); Burbano y Moreno (2018); y, Crespo (2019); consideraron que el primer paso es realizar un diagnóstico territorial, el cual determina las ventajas comparativas para potencializar la región, lo que conlleva a generar desarrollo económico local. En este caso, teniendo en cuenta los direccionamientos de las administración pública, que para el caso de Colombia se halla en cabeza de los alcaldes municipales, quienes postulan esto en sus planes de gobierno y se convierten en transformadores de sociedades.

Como lo planteó en la década de los 80 el autor Vázquez-Barquero (1988), el anterior es un proceso de crecimiento y cambio estructural que, a través de la utilización del potencial de desarrollo del territorio, genera cambios en su entorno. Más aun, en un territorio que vive con temor y con una economía subterránea por sus cultivos ilícitos, pero con la esperanza de planearse un horizonte en el posconflicto.

Cuando la comunidad es partícipe de su propio desarrollo debe tomar en cuenta la planificación territorial para generar cambios en su entorno desde abajo, hacia arriba y abajo; también el concepto de desarrollo endógeno y la postura de la Organización Internacional del Trabajo (OIT), mediante lo establecido por Alburquerque (2004), quienes aseveraron que el desarrollo local, es un proceso que requiere la participación y que fomenta los acuerdos de colaboración entre los principales actores públicos y privados de un territorio, posibilitando el diseño y la puesta en práctica de una estrategia de desarrollo común, con el propósito de aprovechar los recursos y las ventajas competitivas locales; lo que se complementa con la postura de Rodríguez, et al. (2017) sobre esta temática.

En consonancia con el planteamiento realizado por Kisman y Tasar (2014), para la realización de una planificación territorial con el aprovechamiento del desarrollo endógeno, es necesario hacer énfasis en la articulación entre los actores locales: Administración pública, tejido empresarial y la sociedad, que deben realizar un proceso dinámico en torno a ello. A continuación, en el Gráfico II se presenta el proceso en tres líneas principales: Una entrada, que son los insumos del territorio; los productos, que son los beneficiarios; $\mathrm{y}$, con estos se tienen los resultados, enfocados en el bienestar colectivo; todo articulado en los planes de gobierno. 


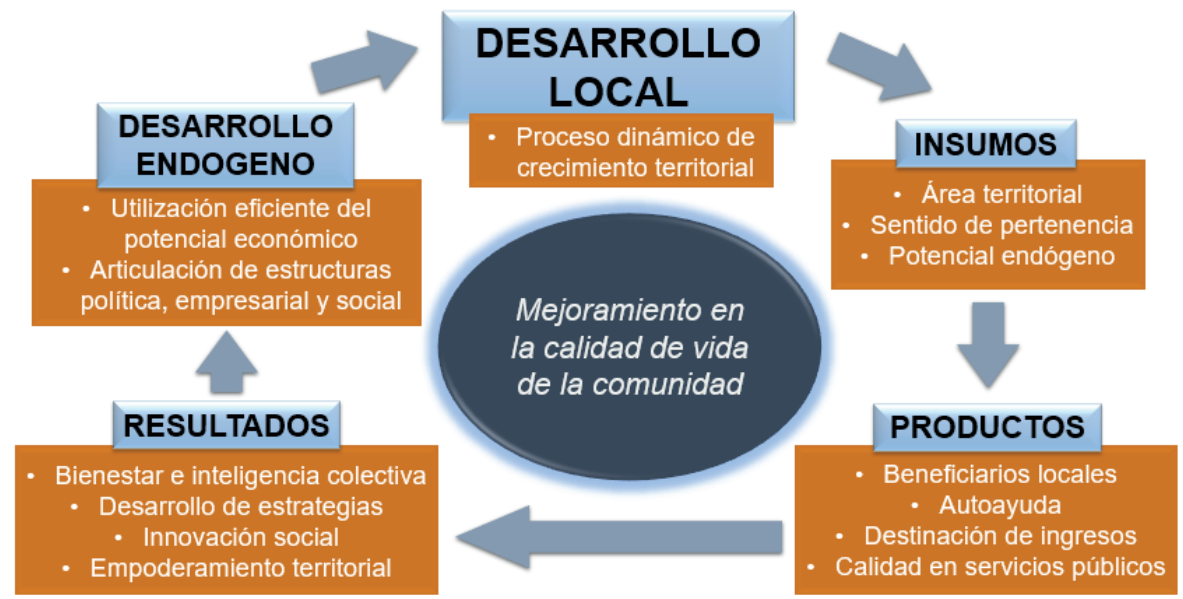

Fuente: Elaboración propia, 2020 con base en Comisión Económica para América Latina y el Caribe (2000); y, Kisman y Tasar (2014).

\section{Gráfico II: Proceso dinámico del desarrollo territorial}

Ahora bien, para el municipio de Corinto, una vez firmado el Acuerdo de Paz en el 2016, como primer paso para la transformación de la región, que por más de dos décadas ha tenido el estigma de ser un territorio epicentro de violencia, está convertirse en un territorio de paz y oportunidades para sus habitantes. Es de mencionar, que el desarrollo local es un camino para esta transformación, tal como lo determinaron Yusoff, Yusof y Arshad (2014); así como Boccella y Salerno (2016), el plan local debe estar preparado y ser concurrente a la luz de las políticas de planificación del gobierno, en el caso de Colombia, se debe hacer referencia al Plan Nacional y a los regionales. Igualmente, para VázquezBarquero y Rodríguez-Cohard (2016), se requiere una sinergia entre los actores locales, lo que complementa la importancia que tiene la comunicación, al permitir un reconocimiento del territorio.

Por su parte, Casey, et al. (2018) consideraron que, en este orden, el primer paso es la realización sistemática de un diagnóstico territorial, que posibilite una planificación adecuada, que al mismo tiempo conlleve a la búsqueda de la competitividad regional; como se explica en el Gráfico III, el factor principal es el trabajo colaborativo entre las entidades y los actores locales, con un empoderamiento de la región, pero en sintonía con la planificación nacional, el eje central es el desarrollo endógeno local. 


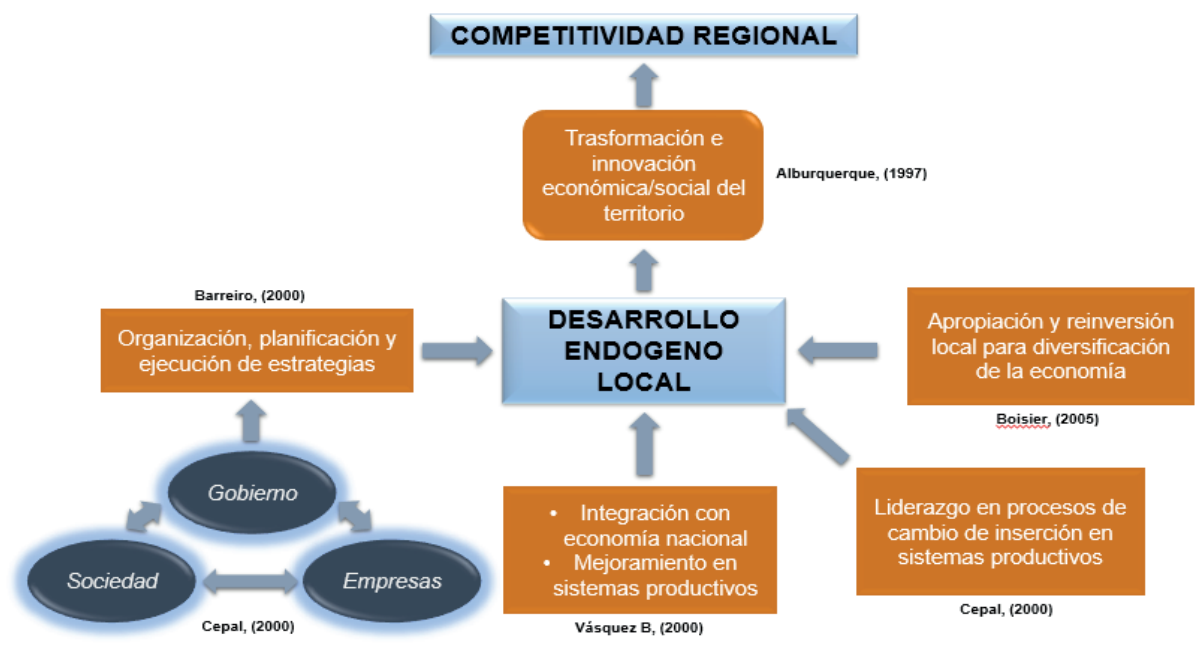

Fuente: Elaboración propia, 2020.

\section{Gráfico III: Proceso para llegar a la competitividad regional desde el desarrollo local}

En este proceso del municipio de Corinto, dentro de los actores locales con un alto conocimiento de la historia del municipio se da el reconocimiento de sus necesidades, en especial en cuanto al fortalecimiento del tejido empresarial, el cual es incipiente y en él se tiene una connotación netamente agricultora, pero por el contrario, no se evidencian industrias establecidas formalmente en la región urbana, como lo establece la página oficial del municipio, donde se indica que "la zona urbana tiene una variedad de establecimientos comerciales, predominando los sitios donde se expenden bebidas alcohólicas y comidas, respecto al comercio de otros artículos es incipiente, predominan los establecimientos comerciales pequeños, familiares" (Comité Local para La Prevención y Atención de Desastres [CLOPAD], 2009, p.8).
De esa manera, se identifica una economía de pequeña escala que no cuenta con un alto apalancamiento financiero ni tampoco con grandes industrias que promuevan el empleo formal dentro de la región; por el contrario, se centra en la parte urbana del municipio, en pequeños almacenes $\mathrm{y}$ en actividades informales, dado que no se cuenta con un registro mercantil de los establecimientos.

En Corinto debe darse la articulación integral entre los actores locales, mencionados en el Cuadro 1, los cuales están llamados a trabajar por el desarrollo local, de modo que esto permita el mejoramiento del sector productivo, que se realicen iniciativas empresariales para generar empleos formales, fortalecer la infraestructura e incentivar la cultura y la educación. 


\section{Cuadro 1}

\section{Actores locales de Corintio}

\begin{tabular}{lcc}
\multicolumn{1}{c}{ Sociedad } & $\begin{array}{c}\text { Tejido } \\
\text { empresarial }\end{array}$ & Administración publica \\
\hline Asociaciones de campesinos sin tierra & Almacenes & Alcaldía municipal de Corinto \\
Zonas de reservas campesinas & & $\begin{array}{l}\text { Unidad Municipal de Asistencia } \\
\text { Cabildo Indígena del resguardo Páez de Corinto }\end{array}$ \\
Asmudescor & Técnica Agropecuaria (Umata), \\
Federación nacional de cafeteros & Secretarías municipales. \\
Representantes de la sociedad & \\
Consejos Comunitarios & \\
Afrocolombianos: La paila y barrios urbanos, el Barranco & \\
y Jagual & \\
\hline
\end{tabular}

Fuente: Elaboración propia, 2020.

En las últimas décadas, los habitantes de Corinto fueron desplazados y aprendieron únicamente a sobrevivir, por lo que se evidencia una desarticulación de su propia realidad, sumado a la imagen de territorio golpeado por el conflicto armado, así que presenta una debilidad con relación al tejido empresarial, el temor de generar empresa, lo que a su vez evidencia falta de liderazgo en el desempeño institucional, la infraestructura, la educación básica, media, superior y de capacitación, la eficiencia de los mercados, la salud, los indicadores medioambientales y la dinámica empresarial asociada con la innovación.

Así pues, con las esperanzas puestas en el posacuerdo, se espera que se potencialicen los territorios por medio del programa Plan para la Paz, el cual, según el Departamento Nacional de Planeación (DNP, 2017), los mecanismos de gestión de la inversión no tienen una apropiación presupuestal ni tienen recursos ciertos, son dinámicas de gestión que se presentan en el marco de acuerdos entre la nación y los territorios. Asimismo, Calle e Isaza (2019) sostienen que: "En momentos de dificultades, la sociedad reacciona y se asocia para aportar soluciones en conjunto" (p.159), como compromiso para la construcción de la paz, que permita alcanzar el desarrollo del territorio.

En ese sentido, el presente estudio se desarrolló en el municipio de Corinto Cauca, en el marco del Acuerdo de Paz que se firmó en el año 2016, con el fin de analizar la perspectiva de desarrollo local, teniendo en cuenta el reconocimiento del territorio a partir de las dimensiones económica, social, administrativa, cultural y medioambiental, desde un enfoque prospectivo y la aplicación del método de análisis estructural.

\section{Metodología}

En la presente investigación se utilizó el análisis estructural, el cual permite realizar el estudio de la región en cuestión para orientarlo hacia un cambio de paradigma, de territorio de violencia a un territorio de paz, desde el enfoque prospectivo; y de esta forma, conocer las variables controlables y reducir la incertidumbre en el futuro.

Asimismo, para el objeto de estudio del municipio de Corinto, se tomaron como fuente de apoyo los planteamientos realizados por Godet (1993), en su manual de prospectiva y estrategia, donde "explora escenarios a futuros", estableciendo que las regiones deben invertir recursos para generar desarrollo local y haciendo énfasis en el cálculo de probabilidades. Según Burbano y Moreno (2015), en las relaciones de la matriz del análisis estructural "lo que interesa es determinar cuáles son las variables o grupos de variables que tienen un mayor valor explicativo acerca del funcionamiento del sistema" (p.250), en este caso, dicho sistema 
se configura como el desarrollo local del municipio de Corinto - Cauca.

Por otra parte, se identificaron las variables clave propias del método, y se debe resaltar que "el objetivo del método es identificar las variables motrices y de enlace, construyendo una tipología de las variables en clasificaciones directas e indirectas" (Burbano y Moreno, 2015, p.251). en ese sentido, para la aplicación de la metodología se desarrollaron tres etapas, como se describen a continuación:

\subsection{Etapa 1: Taller exploratorio}

Para el desarrollo del taller se tomaron en cuenta dos momentos: En primer lugar, la sensibilización y el reconocimiento del municipio a partir de su realidad; en segundo lugar, la organización de grupos elegidos por el actor social, con el fin de seleccionar las variables y hacer el análisis de los efectos y las consecuencias en la región. Este taller finalizó con la socialización y la puesta en común de las variables en las cuales se debe invertir y fortalecer, para que a mediano y largo plazo Corinto sea protagonista de su propio desarrollo. En el Cuadro 2, se exponen las variables elegidas que servirán de insumo para la segunda etapa.

Variables de desarrollo local - Corintio, Cauca

\begin{tabular}{|c|c|c|}
\hline Variable & Indicador asociado & Fuente \\
\hline \multirow[t]{3}{*}{ Geografía } & Extensión territorial & $\begin{array}{l}\text { Fichas de caracterizacion del Departamentoo } \\
\text { Nacional de Planeación. }\end{array}$ \\
\hline & Cartografía municipal & Fichas de caracterizacion del Departamentoo \\
\hline & Población total & Nacional de Planeación y el Plan de \\
\hline \multirow[t]{2}{*}{ Demografía } & Población etnias & Desarrolloo Municipal 2016 -2019 \\
\hline & Población desplazada & \\
\hline Educación & Infraestructura: Colegios & Plan de Desarrollo Municipal 2016 - 2019 \\
\hline Salud & $\begin{array}{l}\text { Cobertura y tasa dee analfabetismo } \\
\text { Regimen contributivo }\end{array}$ & Plan de Desarrollo Municipal 2016 - 2019 \\
\hline $\begin{array}{l}\text { Saneamiento } \\
\text { basico } \\
\text { Vivienda }\end{array}$ & $\begin{array}{l}\text { Regimen subsidiado } \\
\text { Servicios públicos } \\
\text { Hogares déficit }\end{array}$ & $\begin{array}{l}\text { Fichas de caracterizacion del Departamentoo } \\
\text { Nacional de Planeación. }\end{array}$ \\
\hline \multirow[t]{3}{*}{ Turismo } & Recursos naturales & Plan de Desarrollo Municipal 2016 - 2019 \\
\hline & Infraestructura vial & \\
\hline & Conectividad & Plan de Desarrollo Municipal 2016 - 2019 \\
\hline Movlidad & Pasajeros y carga & \\
\hline
\end{tabular}

Fuente: Elaboración propia, 2020. 


\subsection{Etapa 2: Aplicación del análisis estructural}

En esta etapa se revisaron las relaciones entre las variables que se establecieron en la Etapa 1, con el propósito de determinar la importancia de estas en el desarrollo del municipio a futuro. El primer momento de esta etapa, fue la sensibilización y la explicación a los actores sociales sobre la calificación para ingresar los datos a la matriz de influencia/ dependencia, lo que permite evidenciar las relaciones indirectas y los efectos feedback entre las variables, aportando dos tipos de resultados: La clasificación de las variables del sistema territorio, en función de su sensibilidad al entorno (variables internas motrices y dependientes); y la clasificación de las variables de "entorno", en función de su impacto sobre otras variables (externas, motrices y dependientes), lo que se conoce como MIC - MAC, que valora el influjo de cada actor sobre el grupo, tendiendo a criterios de evaluación predeterminados: 0.- Influencia nula; 1.- Influencia débil; 2.- Influencia media; $\mathrm{y}, 3$.- Influencia fuerte.

\subsection{Etapa 3}

Una vez realizada la calificación y el ingreso al MIC - MAC, los resultados se contrastan con información de fuentes secundarias, como son: Planes de desarrollo, rendición de cuentas municipales, datos de planeación departamental y municipal, datos históricos de la violencia (revisión de periódicos) y fichas de territoriales del DNP.

\section{Resultados}

En el análisis estructural y en el resultado de la Matriz de influencia/dependencia (ver Figura I), se tienen las variables población y geografía, las cuales se ubican en el cuadrante superior, y para el municipio de Corinto, que se ubica al suroccidente de la República de Colombia en el extremo norte del departamento del Cauca, sobre las estribaciones de la cordillera central, su estratégica ubicación le imprime un carácter de escenario paisajístico invariable.

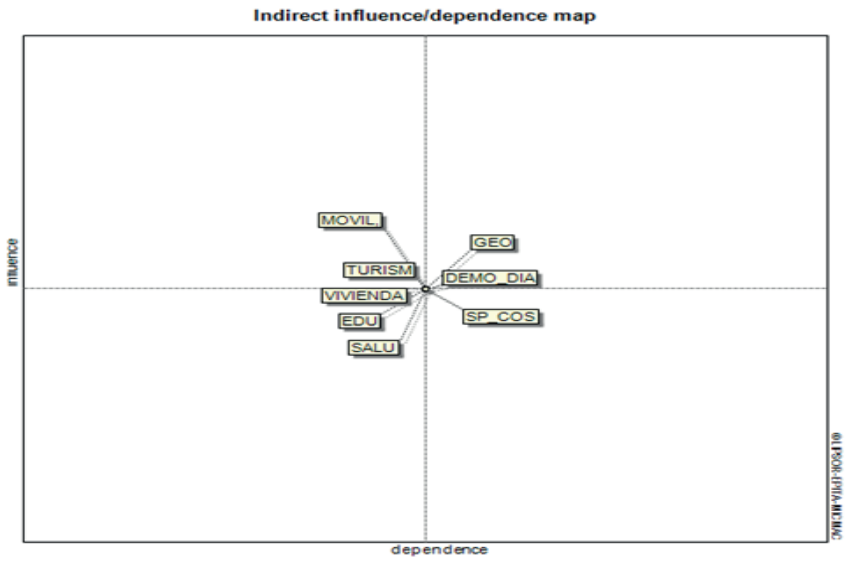

Fuente: Elaboración propia, 2020.

Figura I: Resultados del análisis estructural del desarrollo local Corinto-Cauca 
De acuerdo con la Ley 617 de 2000 (Congreso de la República de Colombia, 2000), Corinto es clasificado como Categoría 6, rural intermedio, tiene una distancia de $118 \mathrm{~km}$ de la ciudad de Popayán, capital del departamento del Cauca, y $65 \mathrm{~km}$ de Cali, capital del departamento del Valle del Cauca, con una población total de 25.440 , con una participación en la zona urbana del 49,3\% y en zona rural de 50,7\% (ver Gráfico IV), también cuenta con un resguardo indígena, posee una población indígena de 8.971 y afrocolombiana de 2.507 (DNP, 2017).

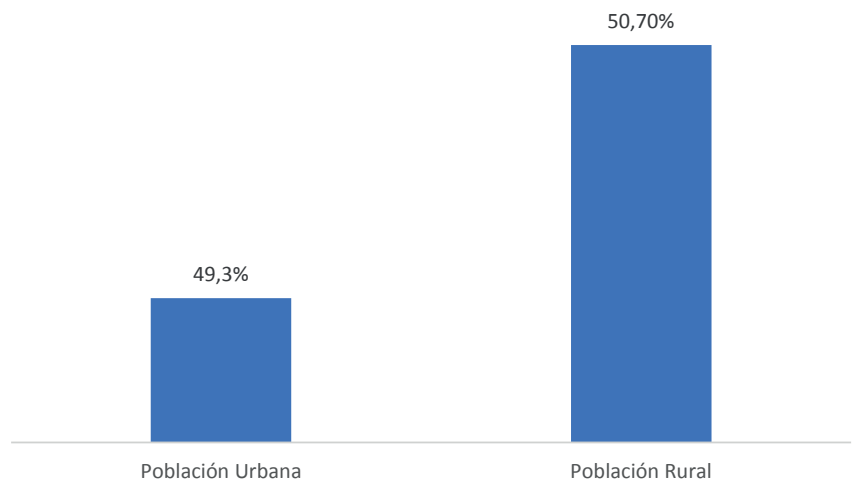

Fuente: DNP (2017).

\section{Gráfico IV: Porcentaje de población urbana y rural}

Es preciso señalar, que en el municipio la economía se basa principalmente en servicios, a pesar de tener potencial para la agricultura, por sus suelos, solo se presentan cultivos transitorios de maíz, café, caña de azúcar, cebolla en rama y café en algunas veredas; pero de acuerdo con el informe de monitoreo de territorios afectados por cultivos ilícitos, se ha incrementado la siembra de estos últimos.

Como puede evidenciarse, en la Figura I se encuentran en la parte superior izquierda las variables de entrada, que son fuertemente motrices y poco dependientes, las cuales determinan el funcionamiento del sistema de desarrollo local de Corinto, en las cuales se tiene: Por un lado, a la movilidad, que es la que dificulta la conectividad, dado que se reconocen las falencias que tiene en la infraestructura en vías secundarias y terciarias; además, la distancia con la capital del departamento del Cauca, el municipio se integra al eje vial de Santander de Quilichao Corintio - Palmira, donde el flujo vehicular es importante así como la conexión que establece con la zona industrial del norte del Cauca. Esta variable es significativa, debido a que en el taller exploratorio la comunidad expresó que se requiere de mantenimiento en las vías y una mejor conectividad, no solo con los principales centros urbanos sino con las veredas.

Por otro lado, la otra variable que se ubica en este cuadrante superior izquierdo (ver Figura I), es el turismo, que tiene importancia después de la firma del Acuerdo de Paz en el 2016, contrastando la percepción de la comunidad en el taller exploratorio que visualizan a Corinto con un potencial para el turismo; también se encuentran las apuestas 
de la administración municipal, como se consagra en el plan de desarrollo en el eje de turismo. "El municipio de Corinto, cuenta con atractivos turísticos que se fortalecen en su belleza natural por su ubicación geográfica y condiciones agroecológicas que parten de una riqueza particular de recursos naturales con paisajes y parajes únicos" (Concejo Municipal de Corinto - Cauca, 2016, p.89).

De igual manera, en el mapeo de los sitios turísticos que se pueden fortalecer e invertir $\mathrm{y}$ que se pueden convertir como una fuente de ingreso y reconocimiento del territorio, se tiene al Parque Central, el Puente de los Esclavos, la iglesia San Miguel Arcángel, la Laguna la Paila, el río La Paila, la Cascada Cominera, la Cascada el Duende, el Museo La Cristalina, el Museo Arqueológico e Histórico de la Cultura Bolo, la Quebrada Seca, pesca deportiva El Edén y el Centro Recreacional Las Pirámides. Igualmente, cuenta con zonas de interés arquitectónico y la riqueza histórica derivada de la época colonial, asociado con las tradiciones ancestral y religiosas de su cultura, que a pesar del conflicto se han logrado conservar.

En el centro de la Figura I, se sitúan las variables que regulan el buen funcionamiento del sistema de desarrollo local, en las cuales se halla la vivienda. Como se determinó en el taller exploratorio, en el municipio se tiene un déficit en vivienda cualitativo del 42,1\%, según el Censo Nacional de Población y de Vivienda del 2018 del Departamento Administrativo Nacional de Estadística (DANE, 2020), esto significa hogares susceptibles de ser mejorados con relación a pisos, hacinamiento, servicios públicos y lugares inadecuados para preparar alimentos, lo que afecta en gran medida la calidad de vida de los habitantes del departamento. Además, con una participación del $10,75 \%$ se encuentra hogares en déficit cuantitativo, aquellos que presentan una falta de habitaciones pertinentes con la cantidad de personas que viven en cada casa, especificado principalmente en falta de paredes (divisiones), cohabitación y hacinamiento no habitable.

En la parte de abajo y a la derecha figuran las variables de salida (ver Figura I), que dan cuenta de los resultados del funcionamiento del sistema de desarrollo local; cabe aclarar que las variables son poco influyentes y muy dependientes. También son variables resultado, las cuales se alinean a indicadores de evolución, que se convierten en metas a mediano y largo plazo; lo cual se alinea al plan de desarrollo municipal, sus apuestas y retos con la región. En este caso, para Corintio se tiene a educación así como salud y saneamiento básico; el análisis de la variable educación es importante para el desarrollo local, tomando en cuenta que para autores como Tapia, et al. (2017); Gambarota y Lorda (2017); y, Burgos (2016), la educación es el motor de desarrollo local y esto se complementa en el Plan de Desarrollo Municipal (Concejo Municipal de Corinto - Cauca, 2016), en el cual reza que:

La educación constituye un aspecto fundamental para el desarrollo integral del hombre y en el medio para transmisión de la cultura a través de valores, normas, costumbres, habilidades, destrezas y actitudes que deben buscar la transformación social y del medio ambiente, a fin de contribuir con el desarrollo de las actividades socio-económicas y culturales en la búsqueda de un mejor bienestar de la comunidad. (p.48)

De tal forma, en los datos de educación para Corinto se observa que no cuenta con instituciones de educación superior, para profesionalizarse los habitantes deben desplazarse a la capital del departamento del Cauca. El municipio cuenta con seis instituciones educativas, tres rurales y tres urbanas, representadas en 46 sedes, un Centro Educativo oficial, dos Centros Educativos privados y una institución para el trabajo y desarrollo humano, lo cual refleja una sesgada capacidad de cubrimiento para el total de habitantes en etapa de aprendizaje. Además, se resalta que aproximadamente un $80 \%$ de la población solo tiene educación primaria, el $32,96 \%$ cuenta con bachillerato y no cuenta con educación superior (ver Tabla 1 ); estas participaciones son similares a las departamentales. 


\section{Tabla 1}

Variable educativa

\begin{tabular}{|c|c|c|c|c|c|c|}
\hline Variable & Consideración & Corinto & Medida & $\begin{array}{l}\text { Despartamento del } \\
\text { Cauca }\end{array}$ & Medida & $\begin{array}{c}\text { Porcentaje de } \\
\text { participación/Corinto }\end{array}$ \\
\hline \multirow{8}{*}{ Educación } & \multirow{2}{*}{ Tasa de analfabetismo } & \multicolumn{5}{|c|}{ Datos 2017} \\
\hline & & 1.849 & $\mathrm{Hab}$ & 81.450 & $\mathrm{Hab}$ & $2,27 \%$ \\
\hline & $\begin{array}{l}\text { Tasa de cobertura } \\
\text { Transición } \\
\end{array}$ & $51,98 \%$ & Est & $52,70 \%$ & Est & $98,63 \%$ \\
\hline & Tasa de cobertura Primaria & $77,74 \%$ & Est & $74,53 \%$ & Est & $104,31 \%$ \\
\hline & $\begin{array}{c}\text { Tasa de cobertura } \\
\text { Secundaria }\end{array}$ & $66,95 \%$ & Est & $69,24 \%$ & Est & $96,69 \%$ \\
\hline & Tasa de cobertura Media & $32,96 \%$ & Est & $43,55 \%$ & Est & $75,68 \%$ \\
\hline & $\begin{array}{c}\text { Tasa de matriculación } \\
\text { oficial }\end{array}$ & $77,08 \%$ & Est & $82,21 \%$ & Est & $93,76 \%$ \\
\hline & Cantidad de sedes activas & 7 & Inst & 556 & Inst & $1,26 \%$ \\
\hline
\end{tabular}

Fuente: Elaboración propia, 2020 con base en datos del Concejo Municipal de Corinto - Cauca (2016).

Como resulta evidente, los servicios públicos son de impacto para la comunidad que habita dentro de un territorio, por ende, es de vital importancia que su cubrimiento esté en un $100 \%$ de cumplimiento. De ese modo, se hace una comparación de los datos obtenidos en el censo realizado por el DANE en 2005, con los datos actuales especificados por el plan estratégico de inversiones de la Empresa Caucana de Servicios Públicos S.A., en donde se especifica directamente el mejoramiento que ha tenido la cobertura de agua potable de un $49 \%$ a un $97 \%$ en el municipio de Corinto, al igual que el cubrimiento en alcantarillado de un $45 \%$ a un asombroso $95 \%$. Desde la Gobernación, se destina un 2,27\% aproximadamente, que es la inversión que obtendrá el municipio de Corinto de acuerdo con su cantidad de habitantes y las obras propuestas para este año (ver Tabla 2).

Tabla 2

Variable servicios públicos básicos

\begin{tabular}{|c|c|c|c|c|c|c|}
\hline Variable & Consideración & Corinto & Medida & $\begin{array}{c}\text { Despartamento del } \\
\text { Cauca }\end{array}$ & Medida & $\begin{array}{c}\text { Porcentaje de } \\
\text { participación/Corinto }\end{array}$ \\
\hline \multirow{11}{*}{ Servicios públicos } & \multirow{2}{*}{ Agua potable (Zona Urbana) } & \multicolumn{5}{|c|}{ Datos 2014 - Plan estratégico de inversiones 2016 - 2019 EMCASERVICIOS } \\
\hline & & $97,00 \%$ & $\mathrm{Hog}$ & $80,73 \%$ & $\mathrm{Hog}$ & $120,15 \%$ \\
\hline & Agua potable (Zona Rural) & $3,00 \%$ & Hog & $24,44 \%$ & Hog & $12,27 \%$ \\
\hline & \multirow{2}{*}{ Agua potable } & \multicolumn{5}{|c|}{ Datos DANE 2005} \\
\hline & & $49,00 \%$ & Hog & $66,00 \%$ & Hog & $74,24 \%$ \\
\hline & \multirow{2}{*}{$\begin{array}{c}\text { Saneamiento básico } \\
\text { (Alcantarillado Zona Urbana) }\end{array}$} & \multicolumn{5}{|c|}{ Datos 2014 - Plan estratégico de inversiones 2016 - 2019 EMCASERVICIOS } \\
\hline & & $95,00 \%$ & Hog & $73,00 \%$ & Hog & $130,14 \%$ \\
\hline & \multirow{2}{*}{$\begin{array}{l}\text { Saneamiento básico } \\
\text { (Alcantarillado) }\end{array}$} & \multicolumn{5}{|c|}{ Datos DANE 2005} \\
\hline & & $45,00 \%$ & Hog & $44 \%$ & Hog & $102,27 \%$ \\
\hline & \multirow{2}{*}{$\begin{array}{c}\text { Inversión en agua potable y } \\
\text { saneamiento básico }\end{array}$} & \multicolumn{5}{|c|}{ Datos 2018} \\
\hline & & 2.746 .402 .413 & COP & 121.000 .000 .000 & COP & $2,27 \%$ \\
\hline
\end{tabular}

Fuente: Elaboración propia, 2020 con base en datos del DANE (2005) y Concejo Municipal de Corinto Cauca (2016). 
Con respecto a los hallazgos de la variable salud (ver Gráfico V), se halló que la mayoría de la población se encuentra afiliado al régimen subsidiado (21.424), al Sistema General de Seguridad Social en Salud (26.243), al régimen contributivo (4.507) y los afiliados a regímenes especiales (312). Este resultado tiene una relación directa con el mercado laboral de la región, que según datos de planeación municipal, la economía de Corintio es considerada como subterránea y con un mercado laboral del $65 \%$ en condición de informalidad, y el trabajo formal, es el que se da en los puestos de trabajo de la administración pública.

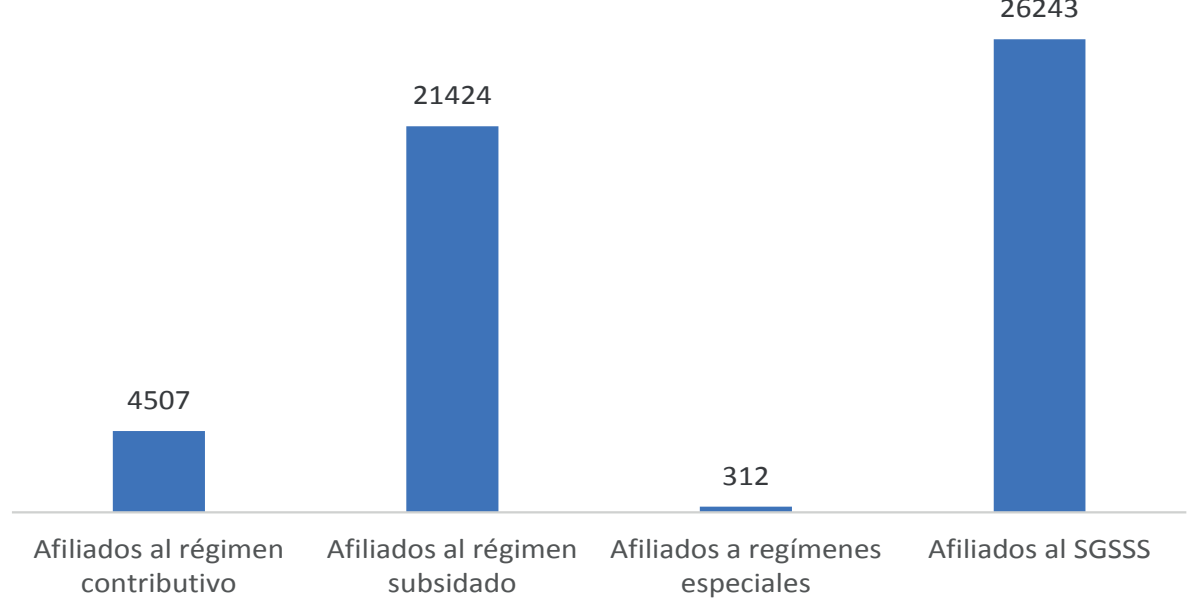

Fuente: DNP (2017).

\section{Gráfico V: Número de afiliados a los distintos regímenes de salud}

Asimismo, se destaca el empleo directo e indirecto que genera la plaza de mercado central; la agroindustria, produce empleo directo en los trapiches paneleros; sin embargo, en el taller exploratorio los actores no tuvieron en cuenta la variable agricultura, dado que en el municipio su principal producción está enfocada en el cultivo ilícito. Como características del municipio, se evidencia que se presenta una tendencia a la emigración hacia la capital del departamento, y uno de los principales motivos es la falta de oportunidades en el mercado, también, como ellos mismo lo expresan, por el temor de la violencia que generó el conflicto armado y que la percepción de confianza hacia las instituciones que el municipio tiene, es baja.

De esta forma, se puede resumir que a partir del Gráfico III y en contraste con la información secundaria (Plan de Desarrollo Municipal), desde las apuestas de sus ejes territoriales y la percepción de los actores locales se tiene que:

a. El turismo y la movilidad, son variables que dinamizarán la economía en los próximos años; en referencia al turismo, 
se tienen tres apuestas por implementar, que serían un plan de desarrollo ecoturístico para el municipio de Corinto, hacer tres rutas ecoturísticas en el municipio; y realizar un estudio así como el diseño de un centro recreativo y parque de agua natural sobre el río La Paila, para lo cual el gobierno garantiza que a mediano y largo plazo esta inversión generará desarrollo local; y estableciendo estrategias de apuestas competitivas que hagan más atractivo el territorio por parte del turismo, lo cual debe ir acompañado de inversión en infraestructura vial en vías primarias, secundarias y terciarias. Asimismo, el gobierno pretende gestionar la ejecución de un proyecto para intervenir 20 $\mathrm{km}$ de la red vial terciaria del municipio.

b. La geografía y la demografía, por su parte, son variables dinámicas de la economía que deben ser potencializadas mediante estrategias de cuidado a la comunidad y velar por que crezcan paulatinamente y de manera acorde con el desarrollo evidenciado dentro del territorio. En relación a la vivienda, la administración a través de proyectos de vivienda urbana y rural busca la disminución de esta brecha, tiene dos retos, gestionar el proyecto de titulación de predios y vivienda nueva para la población vulnerable; $y$, gestionar el regreso de las personas desplazadas.

c. En cuanto a las variables educación, salud y servicios básicos (saneamiento), son consideradas como transversales al sistema $\mathrm{y}$ un buen manejo de estas pueden aportar al desarrollo, por lo que la administración debe realizar apuestas sobre el mejoramiento de ellas. También se espera, en términos de educación, mejorar la calidad; y por otra parte, aumentar la cobertura en salud, a pesar de que se tiene alguna para la población. Finalmente, en los servicios básicos ampliar la cobertura en alcantarillado, energía, conectividad (internet) y gas. Es preciso fomentar todo el desarrollo local, para mejorar el mercado laboral y disminuir la informalidad.

El municipio de Corintio, una vez consolidado el posconflicto en el país, tiene tres apuestas para un mediano plazo, con el propósito de fomentar el desarrollo local a partir de su potencial, las cuales se detallan a continuación

a. Apuesta 1: El desarrollo del turismo, para lo cual el gobierno pretende invertir en dos aspectos: Infraestructura en vías primarias y secundarias, por lo que también debe promoverse la movilidad de pasajeros, también la infraestructura para un desarrollo turístico en relación con hoteles, restaurantes, restauración de partes, senderos ecológicos, aprovechamiento de la biodiversidad; y para ello se requiere capacitación a sus habitantes.

b. Apuesta 2: La educación como motor de desarrollo, por lo cual como apuesta se encuentra el fortalecimiento de la educación, no solo a nivel de los colegios sino también en la cultura, para el cambio de pensamiento hacia un territorio de paz y prosperidad, el perdón de todos los actores involucrados en el conflicto y la creencia en la institucionalidad, que no solo en el municipio está débil, sino que esto también se observa a nivel nacional. Esta variable en un futuro debe dinamizar todo el sistema, con una educación acorde con las necesidades del territorio, de su realidad, que permita apropiarse de todas las ventajas comparativas de la región y convertirlas en competitivas.

c. Apuesta 3: A pesar de que los actores locales no perciben la agricultura como motor de su desarrollo, en la información secundaria se tiene que el municipio posee una vocación agrícola y como apuestas se debe generar transición de los cultivos ilícitos a tradicionales, de modo que la alcaldía va a fortalecer este renglón de la economía con la creación de una zona de desarrollo agropecuario, industrial, turística y de servicios en el municipio, y en articulación con el gobierno nacional, hacer la implementación de un programa de sustitución de cultivos de uso ilícito, mediante el desarrollo de proyectos productivos; también crear una estrategia anual de apoyo de insumos agropecuarios dirigido a pequeños productores.

\section{Conclusiones}

Teniendo en cuenta el análisis estructural realizado para el municipio de 
Corintio - Cauca, el cual tiene un alto potencial para el turismo sostenible, principalmente por la riqueza en la biodiversidad, la cultura y la infraestructura que data de una historia desde la colonia y con la firma del Acuerdo de Paz así como desde el enfoque de desarrollo local endógeno, se tiene una construcción colectiva entre los actores, y se resalta que las apuestas de la administración pública, están enfocadas al cambio de la cultura hacia un territorio de paz y el desarrollo del ecoturismo, pero para lo cual primero es necesario trabajar en el posicionamiento regional, en la confianza de los habitantes en su territorio.

El municipio sobrevivió al conflicto armado y, por ende, hay mucho por hacer. Hasta el año pasado no se vio evidenciado en absoluto una articulación con el plan competitivo nacional, pero actualmente sus habitantes quieren que Corinto no sea visto como municipio de guerra sino como un foco de prosperidad, enseñanza y capacidades productivas. Como lo afirmaron García, et al. (2018), también se requiere de "una transformación cultural donde privan los aspectos humanos de inserción, integración y equidad de las personas que fueron víctimas o victimarios en el conflicto" (p.37). Todo esto dentro del marco del desarrollo para fomentar la competitividad y así generar oportunidades y bienestar para la población.

\section{Referencias bibliográficas}

Alburquerque, F. (2004). El enfoque del desarrollo económico local. Cuaderno de capacitación No. 1. Serie: Desarrollo Económico Local y Empleabilidad. Organización Internacional del Trabajo.

Alburquerque, F. (2005). Rafaela, una experiencia de desarrollo económico local en América Latina. Claves de la Economía Mundial, (1), 331-339.

Alderete, M. V., y Bacic, M. J. (2016). ¿Existe un mejor desarrollo local en los municipios con clúster?: El caso del estado de São Pablo en Brasil. Revista Desarrollo y Sociedad, (77), 53-80.

Boccella, N., y Salerno, I. (2016). Creative economy, cultural industries and local development. Procedia -Social and Behavioral Sciences, 223, 291-296. https://doi.org/10.1016/i. sbspro.2016.05.370

Boisier, S. (2004). Desarrollo territorial y descentralización. El desarrollo en el lugar y en las manos de la gente. Eure, $X X X(90), \quad 27-40$. http://dx.doi.org/10.4067/S025071612004009000003

Burbano, E. L., y Moreno, E. (2015). El desarrollo local desde una visión prospectiva. Revista Virtual Universidad Católica del Norte, (45), 245-257.

Burbano, E. L., y Moreno, E. (2018). Análisis de Conglomerados del Norte del valle del cauca. Caso estudio Cartago, Zarzal y la Unión. Ingeniería Industrial, 39(1), 78-91.

Burgos, R. (2016). El turismo comunitario como iniciativa de desarrollo local. Caso localidades de Ciudad Bolívar y Usme zona rural de Bogotá. Hallazgos, 13(26), 193-214.

Calle, J. S., e Isaza, G. M. (2019). Cooperativismo como compromiso para la construcción de la paz en Colombia. Revista de Ciencias Sociales (Ve), XXV(E-1), 156169. https://doi.org/10.31876/rcs. v25i1.29606

Casey, K., Glennerster, R., Miguel, E., y Voors, M. (2018). Skill versus voice in local development. National Bureau of Economic Research, Working Paper (25022), 1-70.

Comisión Económica para América Latina y el Caribe - CEPAL (2000). Desarrollo económico local y descentralización: 
Aproximación a un marco conceptual.

CEPAL. https://www.cepal.org/es/ publicaciones/31392-desarrolloeconomico-local-descentralizacionaproximacion-un-marco-conceptual

Comité Local para La Prevención y Atención de Desastres - CLOPAD (2009). Documento caracterización de escenarios de riesgos municiio de Corinto Cauca. Gobierno Municipal de Corinto - Cauca. https://1library. co/document/yngr4vjz-documentocaracterizacion-de-escenarios-riesgomunicipio-corinto-cauca.html

Concejo Municipal de Corinto - Cauca (2016). Plan de Desarrollo Municipal: "Nuevas Ideas para la Paz" 20162019. Acuerdo No. 008 de 2016. https://cpd.blob.core.windows.net/ test1/19212planDesarrollo.pdf

Congreso de la República de Colombia (2000). Ley 617 del 6 de octubre del 2000. Diario Oficial No. 44.188. Por la cual se reforma parcialmente la Ley 136 de 1994, el Decreto Extraordinario 1222 de 1986, se adiciona la Ley Orgánica de Presupuesto, el Decreto 1421 de 1993, se dictan otras normas tendientes a fortalecer la descentralización, y se dictan normas para la racionalización del gasto público nacional. https:// colaboracion.dnp.gov.co/CDT/ Inversiones $\% 20 \mathrm{y} \% 20$ finanzas $\% 20$ pblicas/LEY 617_DE_2000.pdf

Crespo, D. (2019). Comparando sistemas de innovación: Una revisión de la literatura sobre Alemania y China. Revista Economía y Politica, (29), 87-104. https://doi.org/10.25097/rep. n29.2019.05

Delgado, J. C. (2018). Dimensión prospectiva del actor local en el talento territorial. Revista de Ciencias Sociales (Ve), XXIV(2), 83-93.

Departamento Administrativo Nacional de Estadística - DANE (2006). Censo
General 2005. https://www.dane. gov.co/index.php/estadisticas-portema/demografia-y-poblacion/censogeneral-2005-1

Departamento Administrativo Nacional de Estadística - DANE (2020). Censo Nacional de Poblacion y Vivienda 2018. https://www.dane.gov.co/index. php/estadisticas-por-tema/demografiay-poblacion/censo-nacional-depoblacion-y-vivenda-2018

Departamento Nacional de Planeación - DNP (2017). Fichas de Caracterización Territorial. https://www.dnp.gov. co/programas/desarrollo-territorial/ Paginas/Fichas-de-CaracterizacionRegional.aspx

Fithri, P., Games, D., Lina, E. C., e Hidayat, R. (2020). Factors influencing SME innovation in local government: The case of SMEs in Nagari. International Journal of Management, 11(4), 221231.

Gambarota, D. M., y Lorda, M. A. (2017). El turismo como estrategia de desarrollo local. Revista Geográfica Venezolana, 58(2), 346-359.

García, J., Durán, S. E., Parra, M. A., y Martínez, H. (2018). Inserción, integración y equidad en el ámbito laboral. Escenario empresarial posconflicto en Colombia. Revista de Ciencias Sociales (Ve), XXIV(3), 3649.

Godet, M. (1993). De la anticipación a la acción: Manual de prospectiva $y$ estrategia. Marcombo, D. L.

Kisman, Z. A., y Tasar, I. (2014). The key elements of local development. Procedia Economics and Finance, 15, 1689-1696.

Proaño, S. A., Quiñonez, E. S., Molina, C. J., y Mejía, O. G. (2019). Desarrollo económico local en Ecuador: Relación entre producto interno bruto y sectores 
económicos. Revista de Ciencias Sociales (Ve), XXV(E-1), 82-98. https:// doi.org/10.31876/rcs.v25i1.29598

Rodríguez, M., Vázquez, A., Sarmiento, A., y Millet, Z. (2017). Renewable energy sources and local development. International Journal of Social Sciences and Humanities, 1(2), 10-19. https://doi.org/10.29332/ijssh.v1n2.31

Santos, R. J. (2018). Blessing and curse. The gold boom and local development in Colombia. World Development, 106(C), 337-355. https://doi. org/10.1016/j.worlddev.2018.02.016

Silva, I., y Sandoval, C. (2012). Metodología para la elaboración de estrategias de desarrollo local. Comisión Económica para América Latina y el Caribe. https://repositorio.cepal.org/ handle/11362/7294

Tapia, E. P., Tapia, S. M., Moscoso, J. L., y Ortiz, H. D. (2017). Economía solidaria: estrategia alternativa para el desarrollo local. Visión Gerencial,
16(2), 313-323.

Vázquez-Barquero, A. (1988). Desarrollo local. Una estrategia de creación de empleo. Pirámide.

Vázquez-Barquero, A., y Rodríguez-Cohard, J. C. (2016). Endogenous development and institutions: Challenges for local development initiatives. Environment and Planning C: Government and Policy, 34(6), 1135-1153. https://doi. org/10.1177/0263774X15624924

Villa, S. I., y Berrocal, J. C. (2019). Aportes de la democracia deliberativa en el contexto del postconflicto colombiano. Revista de Ciencias Sociales (Ve), $X X V(\mathrm{E}-1), \quad 253-263 . \quad$ https://doi. org/10.31876/rcs.v25i1.29613

Yusoff, S. M., Yusof, F., y Arshad, A. F. (2014). An Analysis of Local Plan for Development Control at Local Planning Level in the State of Selangor. Procedia -Social and Behavioral Sciences, 153, 574-584. https://doi. org/10.1016/j.sbspro.2014.10.090 